\title{
INVIRTIENDO EL AULA EN ASIGNATURAS DE GESTIÓN DE EMPRESAS UTILIZANDO DIFERENTES HERRAMIENTAS METODOLÓGICAS
}

\section{FLIPPING CLASSROOM IN BUSINESS USING DIFFERENT METHODOLOGIES}

\author{
Rocio Martinez Jimenez (Universidad de Jaén) ${ }^{1}$ \\ Carmen Ruiz Jiménez (Universidad de Jaén) ${ }^{2}$ \\ Resumen:
}

En los últimos años hemos vivido un cambio importante en el ámbito universitario, que ha provocado que sean necesarias nuevas metodologías docentes en las que el estudiante adquiera un rol mucho más activo y responsable de su propio aprendizaje. En esta línea nos encontramos con un nuevo modelo de enseñanza-aprendizaje como es el aula invertida o flipped classroom (Bergmann y Sams, 2012). Esta metodología supone que el tiempo de clase en el aula se dedica a aplicar, experimentar, indagar, etc.; esto es, a trabajar los niveles más altos de la taxonomía de Bloom. Para ello, el profesorado debe elaborar diferentes prácticas o actividades que sirvan al estudiante para reforzar lo aprendido previamente en casa y que, siguiendo a Santiago y Bergmann (2018) pueden incluirse dentro de, entre otras, las siguientes categorías: enseñanza entre iguales, aprendizaje basado en proyectos, estudios de casos, simulaciones, role-playing, aprendizaje cooperativo y gamificación.

En este trabajo presentamos la valoración realizada por los estudiantes sobre las diferentes herramientas utilizadas al invertir el aula en dos asignaturas relacionadas con la gestión empresarial. Más concretamente, analizamos el grado de interés y diversión que han suscitado en ellos, así como en qué medida cada una de estas técnicas les ha hecho participar más activamente, ha mejorado la comunicación con los compañeros y con el profesorado y ha contribuido a una mayor comprensión de las asignaturas. Los resultados muestran que las tres metodologías mejor valoradas por los estudiantes son: la gamificación, el role-playing y el aprendizaje basado en proyectos.

Palabras clave: Flipped classroom; herramientas pedagógicas; gestión de empresas.

Códigos JEL: A22; I23.

\begin{abstract}
:
In recent years, we have experienced an important change in higher education context, which has led to the need for new teaching methodologies in which students acquire a much more active and responsible role. In this context, we find a new teaching-learning model such as the flipped classroom (Bergmann and Sams, 2012). This methodology assumes that class time in the classroom is devoted to applying, experimenting, researching, etc.; that is, working at the highest levels of Bloom's taxonomy. To do this, the teacher must develop different practices or activities that serve the student to reinforce what was previously learned at home and that, following Santiago and Bergmann (2018) can be included within, among others, the following
\end{abstract}

\footnotetext{
1 rmartine@ujaen.es, Universidad de Jaén.

2 cruiz@ujaen.es, Universidad de Jaén.

Recibido 13 de septiembre de 2019. Aceptado 27 de noviembre de 2019.
} 
categories: peer-to-peer teaching, project-based learning, case studies, simulations, roleplaying, cooperative learning and gamification.

In this paper, we present the students' assessment of the different techniques used when inverting the classroom in two subjects related to business management. More specifically, we analyze the degree of interest and fun they have aroused in them, as well as the extent to which each of these techniques has made them participate more actively, has improved the relationship with peers and with the teacher and has contributed to a greater understanding of the subject. The results show that the three methodologies best valued by the students are: gamification, role-playing and project-based learning.

Keywords: Flipped classroom; pedagogical techniques, business administration.

JEL Codes: A22; I23.

\section{INTRODUCCIÓN}

La educación universitaria ha cambiado mucho en los últimos años. Hoy en día, el profesorado ya no está en el centro del proceso de enseñanza-aprendizaje ni es el único que posee el conocimiento. Al contrario, los estudiantes son los que se sitúan en ese centro y los profesores han de utilizar nuevas metodologías capaces de estimular su creatividad, trabajo en equipo y otras competencias o habilidades de las denominadas soft skills que son las más demandadas hoy en día por la sociedad en general y el mercado de trabajo en particular (Gómez y Muñoz, 2018). Estas competencias han de ser trabajadas en el aula con actividades y herramientas que el profesorado ha debido programar y preparar con antelación y que requerirán que los estudiantes trabajen, investiguen, analicen, etc. De esta manera, el estudiante asume la responsabilidad sobre su propio aprendizaje de una forma más activa, flexible e individualizada. Por su parte, el profesorado también cambia de rol, dedicándose en mayor medida a guiar, orientar y dar retroalimentación a los estudiantes de una manera más personalizada (Tourón y Santiago, 2015). Y es que, cuando el estudiante deja de ser un mero receptor de información para pasar a indagar, experimentar, aplicar y descubrir por sí mismo en un ambiente de trabajo dinámico e interactivo, se consiguen mejores resultados (The Flipped Classroom, 2019).

Este modelo pedagógico se ha denominado flipped classroom, flipped learning o, en español, aula invertida, puesto que considera que el modelo tradicional de enseñanzaaprendizaje se invierte de modo que lo que tradicionalmente se realizaba en el aula pasa a hacerse en casa y lo que los estudiantes habitualmente realizaban en su casa pasa a hacerse en el aula. Se crean así situaciones de aprendizaje creativo y significativo dentro del aula mediante la interacción entre los propios estudiantes y con los profesores (Lundin et al., 2018).

En los últimos años ha aumentado de manera notable el número de experiencias de inversión de aula así como los trabajos publicados (Bishop y Verleger, 2013; O’Flaherty et al., 2015; Lundin et al., 2018) que han mostrado las ventajas o los efectos positivos del aula invertida, tanto desde el punto de vista de los estudiantes como de los docentes. Así, el flipped classroom: aumenta la motivación, el compromiso y la satisfacción de los estudiantes con las clases (Davies et al., 2013; Enfield, 2013; McLaughlin et al., 2013 y 2014; Mason et al., 2013; Sidhu et al., 2014; Gilboy et al., 2015; McCarthy, 2016; Nouri, 2016; Aguayo et al., 2019); supone una mayor asistencia a clase, un mayor aprendizaje y mejores relaciones con los compañeros y con los profesores (Bergmann y Sams, 2012; Chen et al., 2014; Roach, 2014; The Flipped Classroom, 2019); hace a los estudiantes más proclives a métodos de enseñanza más innovadores y colaboradores (Strayer, 2012) y supone un mejor conocimiento de las necesidades específicas de cada estudiante (Fulton, 2012; Roehl et al., 2013). 
Todos estos trabajos vienen a confirmar la idea de que el modelo de aula invertida es un modelo dinámico, que se va enriqueciendo con las distintas investigaciones realizadas. Por tanto, creemos que es importante avanzar en esta línea de investigación y dar a conocer nuevas experiencias desde la perspectiva de los estudiantes, contribuyendo de este modo a la mejora y el perfeccionamiento de dicho modelo en nuestras aulas. Así, este trabajo trata de aportar nueva evidencia en este sentido, analizando la valoración que los estudiantes de dos asignaturas relacionadas con la gestión de empresas hacen de las distintas técnicas o herramientas utilizadas en clase en el marco del modelo de aula invertida.

El trabajo está organizado de la siguiente manera. A continuación, se recoge la revisión de la literatura sobre flipped classroom y las diferentes herramientas metodológicas más utilizadas. El tercer bloque se dedica a describir la experiencia de aula invertida, presentando el contexto de las asignaturas, los participantes y toda la información necesaria sobre el desarrollo de la misma, así como la metodología utilizada para la recogida de la información. Finalmente, se presentan los principales resultados y conclusiones del trabajo.

\section{FLIPPED LEARNING Y METODOLOGÍAS ACTIVAS DE APRENDIZAJE}

Desde que Bergmann y Sams (2012) desarrollaron la metodología de flipped learning, el concepto ha ido evolucionando y hoy en día podemos decir que el aula invertida ofrece mayores oportunidades a los estudiantes para desarrollar un pensamiento crítico e independiente y para mejorar su propio proceso de aprendizaje mediante la colaboración e interacción con sus iguales (compañeros) (O'Flaherty et al., 2015; Flores et al., 2016). Bishop y Verleger (2013) definen el aula invertida como aquella donde se utilizan actividades de aprendizaje interactivas y basadas en grupo dentro del aula y una instrucción individual basada en las TICS fuera del aula.

Así pues, la innovación que introduce este modelo metodológico se encuentra en la separación de dos contextos de trabajo diferentes, aunque conectados cognitivamente: el individual (antes y/o después de clase) y el colectivo (durante la clase).

En el primer contexto, a los estudiantes se les pide que vean una serie de videos sobre distintos aspectos o conceptos teóricos. Estas lecciones audiovisuales tienen la ventaja de estar siempre disponibles para que el estudiante pueda verlas en el momento que quiera e, incluso, poder pararlas y volver a verlas cuantas veces quiera (González-Gómez et al., 2016). Aunque en el aula invertida con mayor frecuencia se hace mención a estos videos, también se pueden utilizar otras herramientas interactivas o materiales, tales como libros, audios y podcasts, blogs, artículos, presentaciones digitales, infografías, líneas de tiempo, mapas mentales, etc. (McLaughlin et al., 2014; Lundin et al., 2018). Lo trabajado en este espacio individual va a proporcionar más tiempo y la capacitación necesaria para afrontar de otra manera el tiempo en el aula.

El segundo contexto es donde se realizan las actividades más interactivas que permitan aplicar y reforzar lo aprendido en casa (Khan, 2012; Martínez et al., 2014; Roach, 2014), trabajando fundamentalmente los niveles más altos de la taxonomía de Bloom (aplicar, analizar, evaluar y crear). La interacción entre los estudiantes está asegurada, espontáneamente, porque es la esencia que distingue al ser humano. Sin embargo, para orientar esa interacción hacia el logro de los objetivos de aprendizaje, el profesorado debe intervenir utilizando distintas herramientas o modalidades de aprendizaje.

En este sentido, encontramos muchos trabajos que recopilan y clasifican las distintas técnicas que el docente puede emplear en el aula (Fernández, 2006; Barkley et al., 2007; Gómez y Muñoz, 2018; Santiago y Bergmann, 2018). A partir de ahí, se trata de que cada profesor analice y elija las más adecuadas para invertir el aula. En esta línea, hemos recopilado aquellas 
que según Fernández et al. (2012) y Santiago y Bergmann (2018), son empleadas en mayor medida al hacer uso de una metodología de aula invertida.

\section{Aprendizaje basado en proyectos y aprendizaje basado en problemas (ABP)}

Estos dos métodos de enseñanza-aprendizaje aparecen en la literatura bajo las mismas siglas en inglés: PBL (Proyect/Problem Based Learning). Esto nos puede hacer pensar que estamos hablando de la misma herramienta. Lo cierto es que, en ambos casos, los estudiantes se sitúan en el centro de la acción educativa teniendo que resolver situaciones a través de su conocimiento, recursos, investigación y reflexión.

Sin embargo el aprendizaje basado en problemas, según McGrath (2002), podemos definirlo como un método de aprendizaje en grupo que usa problemas reales como estímulo para desarrollar habilidades de solución de problemas y adquirir conocimientos específicos. Los problemas, similares a aquellos a los que el estudiante se tendrá que enfrentar en su futuro profesional, son el vehículo para el desarrollo de habilidades relacionadas con la solución de problemas y para la adquisición de los conocimientos específicos.

Por su parte, el aprendizaje basado en proyectos comprende un conjunto de tareas de aprendizaje basada en la resolución de preguntas, que implica al estudiante en el diseño y planificación del aprendizaje, en la toma de decisiones y en procesos de investigación, y que culmina en la realización de un producto final tangible que suele estar vinculado al mundo real y que debe ser presentado ante los demás (Jones et al., 1997). Normalmente, su duración en el tiempo suele ser mayor que la del aprendizaje por problemas, las indicaciones suelen ser más generales, dejando un margen más amplio para la creatividad y la flexibilidad. Además, en el aprendizaje basado en problemas se suele trabajar con grupos más grandes. Otro dato interesante que diferencia estas dos dinámicas está relacionado con su aplicación, puesto que el aprendizaje basado en problemas está particularmente extendido dentro de la medicina, las ciencias de la salud y las ciencias sociales (Hommes et al., 1999; De Graaff y Bouhuijs, 1993), mientras que el modelo del trabajo por proyecto se aplica especialmente a los estudios de ingeniería.

\section{Aprendizaje entre iguales o Enseñanza entre iguales}

El aprendizaje entre iguales es una estrategia creada en 1990 por Eric Mazur, profesor de física de la Universidad de Harvard (Santiago y Bergman, 2018). Esta metodología consiste en el planteamiento por parte del profesorado de preguntas conceptuales de respuesta estructurada. Los estudiantes deben reflexionar sobre la pregunta y responder de manera individual. Una vez que el profesorado ha revisado las respuestas y en función del resultado, los estudiantes discuten sus razonamientos y las respuestas con sus compañeros para volver a aportar al profesorado una nueva respuesta individual. Esta es la parte más importante de la metodología, caracterizada por la interacción entre los sujetos, quienes comentan y discuten sus respuestas. El profesorado revisa las respuestas y decide si la cuestión planteada está suficientemente aclarada o si sigue necesitando más explicación.

Esta técnica se sustenta en la idea de que el nivel de logro inicial se incrementa en la interacción entre iguales. Fundamentalmente supone una mayor participación de los estudiantes, incorpora la revisión continua de los contenidos con una retroalimentación inmediata entre profesores y estudiantes y brinda la posibilidad de identificar dificultades en la comprensión de conceptos claves de la asignatura durante la sesión presencial (Lago et al., 2012). De este modo, asimilan mejor los conceptos y se motivan más para hacer trabajos relativos a estos contenidos (Rodríguez et al., 2012). 


\section{Gamificación}

La gamificación consiste en la utilización de las mecánicas del juego, su estética y sus estrategias para involucrar a la gente, motivar a la acción, promover el aprendizaje y resolver problemas (Kapp, 2012). La importancia de la participación del estudiante en su propio aprendizaje o el feedback inmediato que recibe, son algunas de las razones que justifican la introducción de la gamificación en el aula.

Gracias a los principios del juego se crea una dinámica atractiva que estimula a los estudiantes a desarrollar competencias y habilidades, mejorando notablemente su atención y haciendo más entretenido el proceso de aprendizaje. De este modo, el estudiante aprende jugando, con lo que alcanza una mayor satisfacción en las actividades educativas, lo que aumenta la retención de lo aprendido y le estimula para continuar haciéndolo (Monedero y Castro, 2018). La gamificación puede ayudar a los estudiantes de un modo que no logran alcanzar la mayoría de las formas tradiciones de enseñanza.

\section{Role-playing}

De acuerdo con lo que plantean DeNeve y Heppner (1997), el role-playing o juego de roles se considera en la formación universitaria como una técnica de aprendizaje activo, que posee un creciente potencial de demanda entre docentes y estudiantes. Ésta es una herramienta de aprendizaje en la que los estudiantes asumen y representan un rol asignado de antemano. Normalmente, el profesorado configura la simulación y elige el tema, que enmarcará en una determinada situación. A partir de aquí, se deben configurar las distintas posturas, roles o personajes que pueden asignarse, así como unos determinados contenidos que, finalmente, se representarán en el aula. Esta dinámica requiere, por tanto, una participación activa por parte de los estudiantes.

A través de un estilo similar al juego, el role-playing sirve como modelo simulado y simplificado de situaciones reales. Si se crea un ambiente adecuado, el role-playing ofrece la oportunidad a los estudiantes de asumir papeles o practicar habilidades que, de otra manera, no las ejercitarían por miedo a las consecuencias en la vida real. En un entorno en constante cambio, las dinámicas que acercan al estudiante al mundo real suelen dar un gran resultado. Los juegos de roles, en este sentido, constituyen una estrategia interesante para trabajar diversos contenidos, fomentar el debate y diálogo, despertar el interés, descubrir diferentes puntos de vista, etc.

\section{Estudio de casos}

Según Fernández (2006), el estudio de casos "es una técnica en la que los alumnos analizan situaciones profesionales presentadas por el profesor, con el fin de llegar a una conceptualización experiencial y realizar una búsqueda de soluciones eficaces”. Así, el estudio de casos parte de la elaboración o preparación por parte del profesorado de un caso que suele ser una situación del mundo real, aunque también puede ser inventado. Su elaboración requiere de fundamentos teóricos que el alumnado debe poseer, trabajar, recuperar, etc. e integrarlos en su memoria. Requiere grandes dosis de tratamiento de la información, tanto elaborando como organizando el conocimiento para conseguir los objetivos que han sido fijados de antemano o que van configurándose a medida que avanza el desarrollo del caso. Una vez preparado, se presenta el caso a los estudiantes (bien en sesión presencial o para trabajar de manera individual antes de clase) y el tiempo de clase se dedica a la discusión. De este modo, los estudiantes deben analizar qué ha sucedido, por qué se han tomado determinadas decisiones y cómo podrían mejorar o cambiar las cosas. Si el caso es real, después de una discusión lo más completa y sólida posible, se comparte el final del caso y lo que sucedió realmente. 


\section{Debate}

El debate es una situación por la cual el profesorado compromete a sus estudiantes sobre un determinado tema. Normalmente, los profesores invitan a los estudiantes a prepararse y buscar argumentos a favor y en contra de un determinado tema. Durante el debate los estudiantes no han de adherirse necesariamente a la tesis o antítesis que defienden, puesto que un debate es, ante todo, un ejercicio de lógica y de rigor y no una situación en la que cada uno antepone sus valores personales. El debate y la capacidad de expresar en público nuestra opinión son elementos de especial relevancia en la actualidad y, sobre todo, en el aula, puesto que implica trabajar competencias como: saber hablar, transmitir ideas y opiniones, saber escuchar, compartir posturas o rebatir y aceptar lo que opinan los demás.

Según Santiago y Bergmann (2018), el debate se divide habitualmente en dos categorías: el debate para la comprensión y el debate para la profundización. En cualquiera de estos casos, la planificación de las preguntas es esencial de modo que no se deben hacer preguntas para ver si los estudiantes recuerdan los conceptos, hay que ir más allá y plantear preguntas que lleven a los estudiantes a probar aplicaciones de lo que han estudiado o a analizar lo que han aprendido. En general, los profesores son quienes cierran el debate haciendo la síntesis de los argumentos empleados a favor o en contra del tema tratado.

\section{Indagación}

La indagación, también conocida como aprendizaje por indagación o Inquiry-Based Learning (IBL), se remonta a los años 60. El aprendizaje por indagación tiene como objetivo fundamental involucrar a los estudiantes a través de diversas técnicas y herramientas en su proceso de formación, promoviendo un aprendizaje por descubrimiento (Vidal et al., 2011). En este tipo de aprendizaje el profesorado establece los objetivos formativos y fija el proceso para lograrlos. Esto se traduce en el planteamiento de una cuestión clave o problema que requiere que los estudiantes exploren y descubran. El trabajo posterior en clase consiste en aportar lo que han descubierto, lo que han aprendido y llegar a una conclusión conjunta entre todos los participantes.

Con esta herramienta los estudiantes trabajan sin una dependencia directa del profesorado siendo responsables finales de observar, investigar, consultar fuentes, sintetizar ideas, elaborar informes y presentar sus resultados de forma oral, trabajando múltiples competencias como el pensamiento analítico, la resolución de problemas, la comunicación oral y escrita o el pensamiento reflexivo.

\section{Mapas conceptuales}

Los mapas conceptuales fueron ideados por el profesor estadounidense Joseph Novak, con el objetivo de facilitar la comprensión de la información que se ha de aprender. El mapa conceptual, según Novak y Godwin (1988), es una representación gráfica esquemática de conceptos y sus relaciones. Los conceptos guardan entre sí un orden jerárquico y están unidos por líneas identificadas por palabras (de enlace) que establecen la relación que hay entre ellas. Se caracteriza por partir de un concepto principal del cual se derivan ramas que indican las relaciones y jerarquía entre los conceptos.

Esta herramienta fue diseñada para ser utilizada tanto por los profesores, para organizar contenidos de un tema determinado, como por los estudiantes con objeto de que éstos aprendan a usar un recurso para aprender a aprender dentro de cualquier disciplina.

En su elaboración, el estudiante debe jerarquizar contenidos (los conceptos se disponen por orden de importancia, generalmente los fundamentos se colocan en la parte superior o en el centro de la estructura gráfica), seleccionar conceptos (esto implica capacidad de síntesis puesto que los estudiantes deben recoger lo más significativo del tema) y diseñar visualmente el gráfico 
(codificación visual de los conceptos). De esta forma, el estudiante aprende a organizar el pensamiento, a dar una estructura lógica a un contenido, a identificar conceptos o ideas clave de un texto y a establecer relaciones entre ellos. Todo ello implica el ejercicio del pensamiento reflexivo y el fomento de la creatividad.

\section{DESCRIPCIÓN DE LA EXPERIENCIA DE AULA INVERTIDA Y METODOLOGÍA}

Esta experiencia de aula invertida ha sido aplicada en dos asignaturas de dos grados de la Facultad de Ciencias Sociales y Jurídicas de la Universidad de Jaén: Gestión de Recursos Humanos en $2^{\circ}$ curso del Grado en Finanzas y Contabilidad (FICO) y Organización y Administración de Empresas en $1^{\circ}$ curso del Grado en Relaciones Laborales y Recursos Humanos (RRLL). Ambas asignaturas tienen una carga docente de 6 créditos (60 horas de presencialidad) y se han impartido en el primer semestre del curso 2018-2019³.

Todo cambio en la metodología docente conlleva, en primer lugar, dedicar un tiempo a la planificación y programación del proceso. De este modo, las profesoras que decidimos invertir nuestras aulas, nos reunimos al comienzo del cuatrimestre para organizar las asignaturas y la dinámica de clase, trabajando especialmente la selección de recursos y estrategias tanto para la parte grupal (en el aula) como para el trabajo fuera del aula.

A la hora de elegir las herramientas para trabajar en el espacio grupal tuvimos en consideración tanto las recomendaciones de distintos estudios como nuestra propia experiencia previa. En este sentido, Moya y Soler (2018) señalan que aplicar una misma metodología prolongada en el tiempo no arroja mejores resultados en términos de un mejor aprendizaje. Y es que parece que la mejor opción para que perdure en el tiempo el impacto favorable de los nuevos modelos metodológicos en el proceso de aprendizaje, es la combinación de varias herramientas o modelos de aprendizaje. A su vez, Fernández (2006) asegura que no hay conclusiones definitivas en relación a si unos métodos son mejores que otros para la asimilación de los contenidos. Parece, más bien, que cada método cumple mejor unas funciones o fases del proceso de enseñanza-aprendizaje que otras. Por tanto, todo esto parece indicar que el mejor método es, en realidad, una combinación de métodos. Nuestra experiencia en este sentido también corrobora esta idea. Los estudiantes se aburren si siempre se trabaja con las mismas herramientas. Además, debemos ser conscientes de que hay herramientas que son más adecuadas que otras en función de los contenidos y las competencias que se quiera trabajar. En definitiva, podemos afirmar que no existen "recetas mágicas" y que cada profesor o profesora deberá configurar su propia “caja de herramientas” más adecuada.

De este modo, hemos de aclarar que, lógicamente, no hemos utilizado las mismas herramientas en las dos asignaturas por igual, puesto que éstas debían estar adaptadas a los contenidos, las competencias y los resultados de aprendizaje que se esperaba de cada una de ellas. Así, las técnicas que se han utilizado en las dos asignaturas han sido: enseñanza directa del profesor, gamificación, enseñanza entre iguales, aprendizaje basado en proyectos, indagación y estudio de casos. Sin embargo, sólo en el caso de la asignatura Gestión de Recursos Humanos se ha hecho uso del role-playing y del aprendizaje basado en problemas, mientras que en la asignatura Organización y Administración de Empresas se han utilizado los mapas conceptuales y los debates.

Una vez planificado y programado el proceso, comenzamos el trabajo con los estudiantes. La primera toma de contacto con ellos estuvo dedicada a presentar este nuevo modelo

\footnotetext{
${ }^{3}$ Las dos autoras eran las responsables únicas de la docencia de las dos asignaturas de manera independiente, esto es, cada una de ellas impartía sólo una de las dos asignaturas analizadas en este estudio.
} 
metodológico. Creemos que esto era especialmente importante puesto que el aula invertida supone un cambio de roles tanto del profesorado como de los estudiantes y es conveniente que este aspecto quede claro desde el principio, especialmente si los estudiantes no han tenido conocimiento de esta metodología, como ha sido nuestro caso. Ellos deben asumir nuevos compromisos y nuevos roles y deben desarrollar nuevas competencias diferentes a las acostumbradas en el sistema tradicional. Por ello, durante la primera sesión de clase explicamos en qué consiste el aula invertida, para qué nos puede ser útil y cómo la vamos a poner en práctica. Igualmente, presentamos el plan de trabajo y las herramientas con las que se iba a trabajar, antes, durante y después de clase.

En definitiva, se explicó a los estudiantes el cambio metodológico que se iba a realizar en las asignaturas, comentándoles la forma de trabajar tanto en el espacio individual como en el grupal. Igualmente, describimos que la evaluación de las asignaturas sería de tipo formativo y sumativo, esto es, que no sólo se tendría en cuenta el examen teórico final (que representa un 50 por cien de la calificación) sino que, además, se valorarían todas las actividades a realizar tanto fuera como dentro de clase (50 por cien), recibiendo un feedback continuo. De esta forma, en la calificación final de las asignaturas se tenían en cuenta todas las herramientas metodológicas programadas, cada una de ellas con su correspondiente rúbrica de evaluación.

El trabajar con aula invertida se planteó desde el primer momento con carácter voluntario puesto que éramos conscientes de que determinadas circunstancias personales de los estudiantes (cuestiones laborales, cuidado de familiares o dependientes a su cargo, etc...) podían influir en la carga de trabajo fuera y dentro del aula y esto podría afectar al grado de compromiso con esta metodología ${ }^{4}$. Por tanto, para quienes no podían trabajar de esta manera, se les ofreció la posibilidad de estar tutorizados durante el cuatrimestre y ser evaluados siguiendo el sistema tradicional con un único examen final teórico-práctico.

A partir de ahí, la dinámica que hemos seguido en cada una de las sesiones de clases ha sido la siguiente:

a) Antes de clase: para cada unidad, hemos elaborado y facilitado el acceso a "mini contenidos de formación" en formato multimedia (como videos o presentaciones con diapositivas) y en formato papel (textos, infografías, mapas mentales, etc.).

b) Durante la clase: en clase se ha dedicado entre diez y quince minutos a presentar los objetivos y estructura de cada sesión concreta y a revisar el contenido teórico necesario para el desarrollo de dicha sesión, incidiendo especialmente en las dudas o aspectos que no hubieran quedado claros con el material facilitado. A continuación, se comenzaba con la aplicación de las distintas herramientas de aprendizaje comentadas anteriormente.

c) Después de clase: el tiempo posterior a la clase se dedicó a que los estudiantes asimilaran e interiorizaran los principales conceptos de cada tema, con un trabajo autónomo por su parte. Así, este tiempo se refería fundamentalmente a la elaboración de trabajos, esquemas, comentarios, revisiones de los principales conceptos y verificación de lo aprendido.

Se ha trabajado con grupos relativamente reducidos, lo que ha facilitado mucho la puesta en práctica de esta metodología. Concretamente, los estudiantes que han trabajado en estas dos

\footnotetext{
${ }^{4}$ McNally et al. (2017) consideran que hay dos grupos de estudiantes (los “flip endorsers” y los “flip resisters”) que pueden tener una aptitud diferente hacia el trabajo con este tipo de metodología y preferir, en el segundo caso, la tradicional clase estilo lección magistral. En nuestra experiencia, los estudiantes que no quisieron trabajar con la metodología flipped (4 de 33 en FICO y 11 de 46 en RRLL) más que por ser reacios a esta nueva forma de trabajar, optaron por el examen final porque no podían asistir con regularidad a las clases, al tener otras responsabilidades profesionales o familiares que atender en ese horario.
} 
asignaturas han sido 63: 28 en el Grado de FICO y 35 en el Grado de RRLL.Para poder obtener los resultados sobre la percepción de los estudiantes de las distintas herramientas utilizadas en esta experiencia, decidimos utilizar un cuestionario ad-hoc, voluntario y anónimo. Para la elaboración del cuestionario tomamos como referencia los aspectos valorados en los trabajos de Santiago y Bergmann (2018) y Aguilera et al. (2017). Así, elaboramos un cuestionario que incluía cinco preguntas de valoración de cada herramienta en base a diferentes aspectos o ítems: en qué medida ha resultado interesante y divertida; cómo ha ayudado a participar más en clase y a comunicarse más y mejor con sus compañeros y con la profesora; $y$, finalmente, si ha ayudado a la mejor comprensión del contenido de la asignatura.

El cuestionario fue previamente validado por expertos, realizando un pretest con 20 personas entre compañeros y estudiantes colaboradores y consistía en una escala Likert que iba desde el 1 (muy en desacuerdo) hasta el 5 (muy de acuerdo).

La tasa de respuesta a este cuestionario fue muy alta, puesto que recibimos un total de 58 cuestionarios válidos (un 92.1 por cien del total). El reparto de dichos cuestionarios entre asignaturas y titulaciones es más o menos equitativo (24 cuestionarios en FICO y 34 en RRLL).

\section{RESULTADOS}

Comenzando por el grado de interés y diversión que para los estudiantes supuso cada técnica, los resultados que aparecen en los cuadros 1 y 2 muestran que no se produce la misma valoración en ambas asignaturas. Así, los estudiantes de FICO consideran que la gamificación ha sido la técnica que mejor ha cumplido ambos elementos, seguida muy de cerca por el roleplaying. En el primer caso, lo que se utilizó fue la aplicación Kahoot! para realizar tests de evaluación de cada unidad, a la vez que se jugaba a modo de "trivial” y en el segundo caso se hizo una simulación de roles (como una especie de teatro) donde aprendieron cómo comportarse (y cómo no) en una dinámica de selección de personal. Sin embargo, en RRLL el aprendizaje basado en proyectos ha sido considerado más interesante y divertido por los estudiantes. En este caso se trataba de un proyecto a desarrollar durante el cuatrimestre en el que los estudiantes debían diseñar un puesto de trabajo aplicando los conocimientos vistos en cada unidad. La segunda herramienta más divertida para ellos ha sido la gamificación (que igual que en el caso de los estudiantes de FICO se ha trabajado con la aplicación Kahoot! para evaluar la asimilación de contenidos de cada uno de los temas) y la más interesante el estudio de casos, basados en casos reales que fueron seleccionados por los propios estudiantes en función de sus preferencias. 


\section{CUADRO 1. INTERÉS Y DIVERSIÓN DE LAS TÉCNICAS PARA LOS ESTUDIANTES DE FICO}

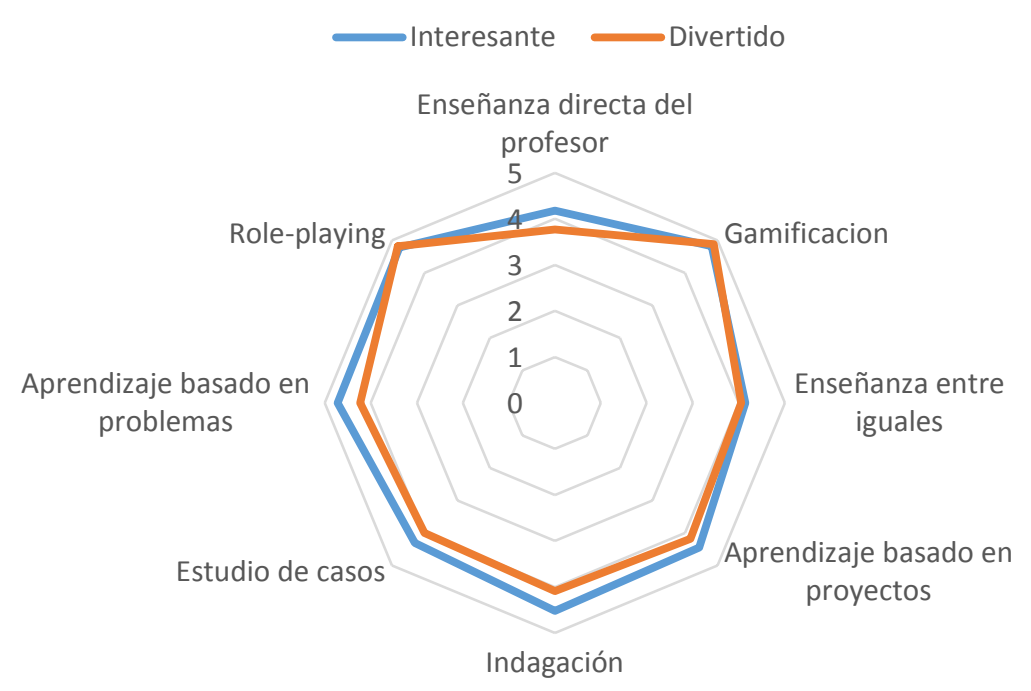

Fuente: elaboración propia.

\section{CUADRO 2. INTERÉS Y DIVERSIÓN DE LAS TÉCNICAS PARA LOS ESTUDIANTES DE RRLL}

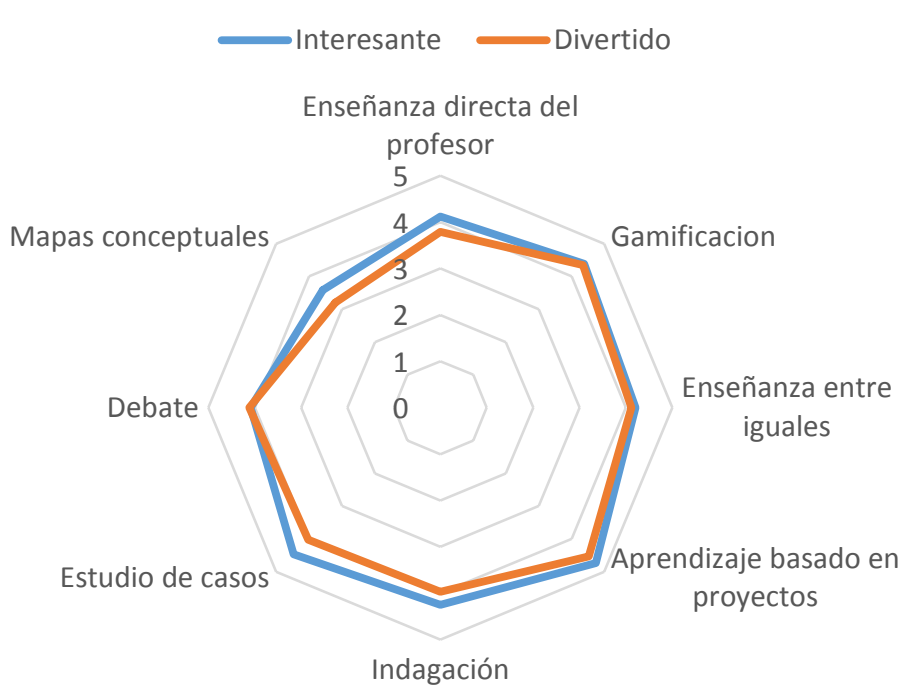

Fuente: elaboración propia.

En cuanto a la mejora de la participación en clase y la comunicación con compañeros y con la profesora, tal y como observamos en los cuadros 3 y 4, vuelve a haber diferentes valoraciones en las dos asignaturas. Así, para los estudiantes de FICO, la herramienta que más favorece la participación y la comunicación es el role-playing, seguido de la enseñanza entre iguales. En RRLL, sin embargo, vuelve a aparecer el Aprendizaje basado en proyectos como la 
herramienta más valorada en los dos aspectos. Como segunda herramienta mejor valorada en relación a la participación le sigue el estudio de casos empatado con la enseñanza entre iguales. $\mathrm{Y}$ en lo referente a la mejora de la comunicación entre compañeros y profesora, el debate y la enseñanza directa de la profesora han sido las herramientas mejor valoradas después del ABP.

\section{CUADRO 3. PARTICIPACIÓN Y COMUNICACIÓN PARA LOS ESTUDIANTES DE FICO}

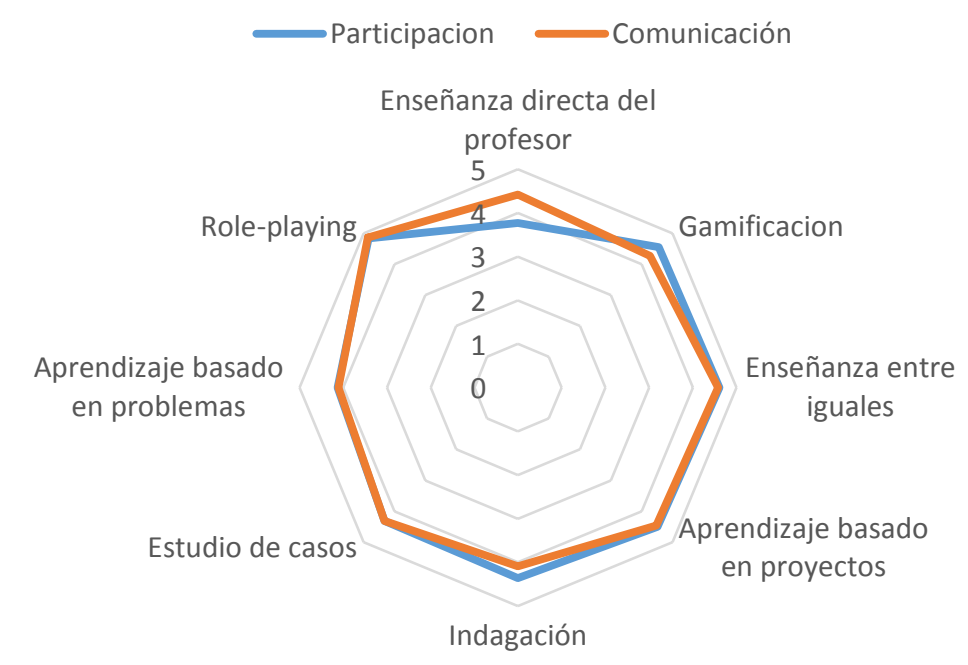

Fuente: elaboración propia.

\section{CUADRO 4. PARTICIPACIÓN Y COMUNICACIÓN PARA LOS ESTUDIANTES DE RRLL}

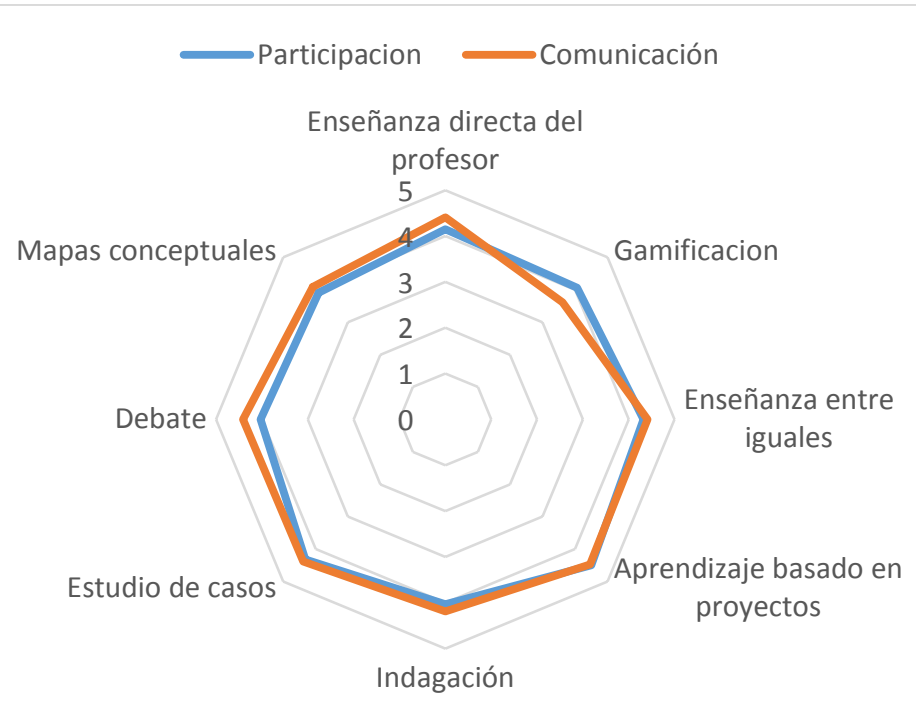

Fuente: elaboración propia. 
Finalmente, si analizamos el grado de comprensión de contenidos que cada herramienta ha supuesto para ellos, podemos comprobar en los cuadros 5 y 6, que en el caso de FICO vuelven a aparecer la gamificación, seguida del role-playing. En cuanto al grupo de RRLL, se produce un empate entre el ABP y el estudio de casos y le sigue muy de cerca la enseñanza directa del profesor.

\begin{tabular}{|c|c|c|}
\hline CUADRO 5. COMPRENSIÓN PARA LOS ESTUDIANTES DE FICO \\
\hline Aprendizaje basado en \\
problemas
\end{tabular}

GRÁFICO 6. COMPRENSIÓN PARA LOS ESTUDIANTES DE RRLL

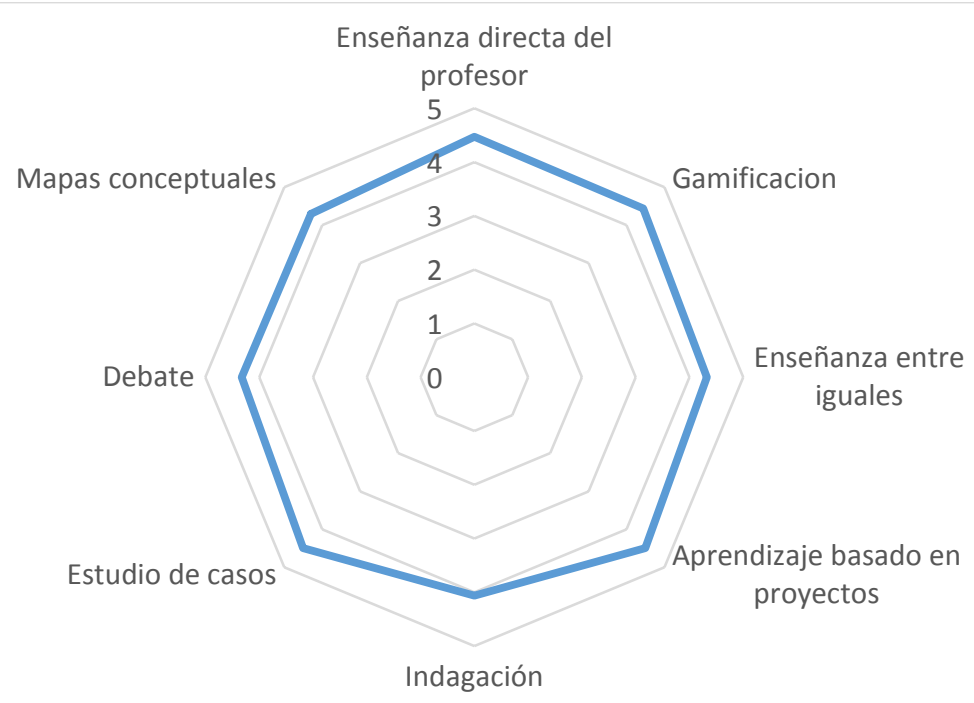

Fuente: elaboración propia. 
Para completar el estudio, realizamos una comparación de medias entre las dos asignaturas, utilizando la prueba U de Mann-Whitney para dos muestras independientes. Dicho test nos ofrece resultados significativos $(\mathrm{p}<0.05)$ para las siguientes herramientas y aspectos valorados: gamificación (en lo que se refiere a interés, diversión, comunicación y comprensión); indagación (en lo referente a interés) y enseñanza directa (en lo referente a comprensión). Así pues, los resultados muestran que existen diferencias significativas en estos seis aspectos puesto que los estudiantes de FICO lo valoran en todos los casos significativamente mejor que los estudiantes de RRLL, tal y como se puede observar en los valores recogidos en la tabla 1.

\section{TABLA 1. RESULTADOS SIGNIFICATIVOS DEL ESTUDIO COMPARATIVO DE MEDIAS}

\begin{tabular}{|l|c|c|c|}
\hline \multicolumn{1}{|c|}{ Aspecto evaluado } & Medias & $\begin{array}{c}\text { Desviaciones } \\
\text { típicas }\end{array}$ & Significación \\
\cline { 2 - 5 } & FICO: 4,82 & FICO: 0,501 \\
RRLificación interesante & FICO: 4,38 & 0,022 \\
& RRLL: 4,35 & $\begin{array}{c}\text { FICO: 0,448 } \\
\text { RRLL: 0,981 }\end{array}$ & 0,007 \\
\hline Gamificación divertido & FICO: 4,27 & FICO: 1,120 & 0,039 \\
\hline Gamificación comunicación & FICO: 4,83 & FICO: 0,388 & 0,032 \\
& RRLL: 4,44 & RRLL: 0,824 & \\
\hline Gamificación comprensión & FICO: 4,52 & FICO: 1,082 & 0,025 \\
& RRLL: 4,25 & RRLL: 0,718 & \\
\hline Indagación interesante & FICO: 4,74 & FICO: 0,689 & 0,031 \\
& RRLL: 4,47 & RRLL: 0,615 & \\
\hline Enseñanza directa comprensión & \multicolumn{3}{|l}{} \\
\hline
\end{tabular}

\section{DISCUSIÓN DE RESULTADOS Y CONCLUSIONES}

En los últimos años hemos podido observar una desmotivación generalizada en los estudiantes que dejan de asistir a clase y que consideran que la típica lección magistral prácticamente no les aporta ningún valor. En este sentido, se ha demostrado que la nueva metodología de enseñanza-aprendizaje denominada flipped learning supone una participación más activa de los estudiantes que redunda también en una mayor motivación, un mayor interés por la asignatura y un mejor rendimiento académico (Fortanet et al., 2013; Sáez et al., 2014; Urbina et al., 2015; Prieto et al., 2016; Aguilera et al., 2017). Esto fue lo que nos animó a desarrollar esta experiencia de aula invertida en dos asignaturas relacionadas con la gestión de empresas (Gestión de Recursos Humanos y Organización y Administración de Empresas).

Invertir el aula supone realizar una programación de la materia para ver cómo se van a trabajar los distintos conceptos, competencias y resultados de aprendizaje. Supone un cambio de chip y un replanteamiento de las actividades que se realizan tanto en el espacio individual como grupal. Podríamos decir que el flipped learning es algo así como el sistema operativo del aula que se nutre de una serie de aplicaciones (herramientas metodológicas) que tienen que darle sentido al modelo. Por eso, es fundamental dedicar un tiempo a planificar y decidir qué herramientas metodológicas se van a utilizar porque, como ya hemos señalado, no hay una única herramienta que resulte adecuada para todas y cada una de las asignaturas e, incluso, para cada uno de los contenidos o unidades que se quieran trabajar. De esta forma, debemos tener 
muy claro cómo utilizamos cada espacio en este contexto para que la interrelación de todas ellas suponga un conjunto sólido y coherente que aporte valor al estudiante y a toda la clase.

Así, en las dos asignaturas en las que hemos invertido el aula, se ha trabajado con algunas de estas herramientas más habituales, tales como el estudio de casos, la gamificación, el aprendizaje basado en proyectos, etc. pero también se han adaptado algunas más específicas que encajaban mejor en cada caso como, por ejemplo, el role-playing en el caso de Gestión de Recursos Humanos o el debate en el caso de Organización y Administración de Empresas.

La valoración que han realizado los estudiantes de esta experiencia demuestra que no existe una única herramienta que sea evaluada como la mejor en todos los aspectos: interés, diversión, participación, comunicación y comprensión. No obstante, gamificación, roleplaying, aprendizaje basado en proyectos y estudio de casos han sido las herramientas que mejores valoraciones han recibido de la mayoría de los estudiantes.

Más concretamente, por lo que se refiere a la diversión, la gamificación ha sido una de las herramientas mejor valoradas. Esto fue constatado por las propias profesoras a lo largo del cuatrimestre puesto que, efectiva y curiosamente, observábamos cómo el hecho de utilizar la herramienta Kahoot! hacía las sesiones de evaluación más divertidas. Utilizar las dinámicas de juego en clase hace que los estudiantes se diviertan y aprendan jugando, reteniendo así en mayor medida los contenidos trabajados.

En cuanto a lo interesante que les ha resultado cada una de las herramientas, hemos de decir que para los estudiantes de FICO, además de repetirse la gamificación, el role-playing ha sido la herramienta mejor valorada, mientras que en el caso de RRLL se trata del ABP. La razón de dicha valoración puede estar en que a través de estas técnicas los estudiantes se acercan a los problemas reales de empresas que les interesan, que les atraen, tratando, en el caso del ABP, de analizar y elegir entre alternativas reales, o bien en el caso del role-playing simulando un proceso de selección de personal.

Otros dos aspectos valorados han sido la mejora de la participación en clase y la mejora de la comunicación con compañeros y con la profesora. En este punto, los estudiantes de FICO vuelven a valorar el role-playing como la herramienta que mejor ha conseguido estos objetivos, seguida muy de cerca de la enseñanza entre iguales. Estos resultados son coherentes con la propia puesta en marcha de estas herramientas, puesto que en ambas ellos son los verdaderos protagonistas, limitándose el profesor a ser un mero observador-evaluador. Los estudiantes deben buscar la información, prepararla, presentarla y explicarla a los compañeros. Por tanto, obviamente, ellos han identificado estas herramientas como las más participativas y en las que se han tenido que comunicar en mayor medida tanto con los compañeros como con la profesora.

En el caso de RRLL, además del ABP, el debate y el estudio de casos han sido las técnicas mejor valoradas. Estos resultados también son coherentes si tenemos en cuenta que el ABP se ha trabajado con grupos de 4-5 estudiantes, lo que ha permitido que haya una mayor interacción y comunicación entre los compañeros. Por su parte, el debate es una técnica que por sí sola invita a la comunicación puesto que los estudiantes deben saber hablar y transmitir sus propias opiniones, defenderlas e incluso hacer ver a los compañeros otras perspectivas del tema tratado. Y en el estudio de casos, el tiempo en el aula ha estado dedicado fundamentalmente a la discusión, al análisis de la situación, de las alternativas... lo que fomenta necesariamente la comunicación, la participación y la interacción entre todos.

Finalmente, también hemos valorado cuál o cuáles de las técnicas utilizadas les ha ayudado a comprender mejor los contenidos. En el caso de FICO aparece nuevamente la gamificación, lo que resulta lógico y esperable puesto que siempre se hacía una reflexión y feedback después de cada pregunta para comprender los fallos y la respuesta correcta. De hecho, algunos de los estudiantes nos comentaron que el Kahoot! les había ayudado a comprender e 
interiorizar mejor los principales conceptos de las asignaturas. Sin embargo, nos llama la atención que la segunda herramienta mejor valorada en este aspecto es el role-playing, que no es una herramienta que pretendiera realmente ayudar a comprender unos conceptos, sino que más bien, se orientaba a trabajar una serie de competencias clave. En este sentido, creemos que se ha podido producir una valoración más positiva influenciada por la valoración del resto de los aspectos.

En el caso de RRLL vuelven a aparecer el ABP y el estudio de casos como las herramientas mejor valoradas en cuanto a la facilitación de la comprensión de los contenidos. Debemos recordar que el proyecto en el que los estudiantes han trabajado durante el cuatrimestre se basaba en el diseño de un puesto de trabajo. Para esto debían tener en cuenta conceptos básicos de cada uno de los temas de la asignatura. Por esta razón, creemos que la revisión conjunta de contenidos les ha llevado a una mejor comprensión de la asignatura. Y por lo que se refiere al estudio de casos, como hemos explicado anteriormente, los estudiantes buscaban información sobre aquellas empresas que más les interesaba estudiar. Ellos han podido elegir y, de esta manera, han trabajado más motivados. Por esta razón, creemos que el estudio de casos ha sido una técnica que les ha facilitado la comprensión de la asignatura.

Por otra parte, algunas de las valoraciones que más nos han sorprendido son, en primer lugar, la puntuación menos favorable que han recibido los mapas conceptuales, a pesar de que algunos estudios (Novak y Gowin, 1988; Novak y Cañas, 2006; Murga-Menoyo et al., 2011; Pontes, 2012) han mostrado su utilidad y relevancia para el aprendizaje. En segundo lugar, podemos destacar el hecho de que la gamificación no haya sido la más valorada en cuanto a facilitar la comprensión de los contenidos por parte de los estudiantes de RRLL, teniendo en cuenta que la forma de trabajar esta técnica ha sido la misma en los dos grados. Y, por último, esperábamos que la enseñanza directa del profesor fuera la herramienta que los estudiantes consideraran que mejor les había ayudado a comprender la asignatura y, sin embargo, no ha sido así (en el caso de RRLL ha tenido una valoración algo más elevada, ocupando el segundo puesto, pero en FICO queda un poco más abajo).

Todas estas valoraciones nos llevan a darnos cuenta de que las nuevas generaciones que se están incorporando a nuestras aulas (mucho más preparadas tecnológicamente y con otras inquietudes y valores) necesitan herramientas mucho más activas y que les demanden un rol más participativo, de manera que pongan en práctica los niveles más altos de la taxonomía de Bloom. Pero también somos conscientes, a raíz de nuestra experiencia, de que ni podemos prescindir completamente de esta enseñanza directa ni es fácil invertir el aula y utilizar metodologías activas. Ello se debe a que esta nueva forma de trabajar supone un importante esfuerzo y mucho trabajo tanto para el profesor como para los estudiantes. La mayoría de nuestros estudiantes no han trabajado nunca con este tipo de metodologías activas. Y esto requiere un período de adaptación a una nueva forma de trabajo y de organización de los tiempos tanto fuera como dentro del aula. Quizá ésta pueda ser la razón por la cual algunos de nuestros estudiantes no hayan estado dispuestos a trabajar con esta metodología y hayan elegido preparar la asignatura de forma autónoma.

Aun así, la experiencia merece la pena por los resultados conseguidos. Por nuestra parte hemos afrontado esta innovación con mucho entusiasmo y responsabilidad. Y por parte de nuestros estudiantes, hemos conseguido una mayor implicación e interés por los contenidos, ha mejorado su nivel de asistencia a clase hasta el último día y ha habido un gran cambio tanto en su actitud como en el clima de trabajo en el aula.

Para finalizar, hemos de comentar que somos conscientes de que una limitación de este estudio es el hecho de que las docentes eran diferentes en las dos asignaturas, lo que puede afectar a los resultados obtenidos. Igualmente, reconocemos que los resultados de este estudio no son generalizables debido al reducido número de estudiantes que han participado en el 
mismo. En este sentido, como línea futura de trabajo nos planteamos replicar este mismo estudio en nuevos cursos incluyendo distintas asignaturas y titulaciones. Del mismo modo, otra línea futura de trabajo sería realizar un estudio cualitativo (mediante entrevistas en profundidad) con los estudiantes para conocer sus opiniones sobre las diferentes herramientas. Esto nos permitiría obtener más información sobre por qué una determinada herramienta resulta divertida, interesante, etc. lo que permitiría discernir si esta valoración es debida únicamente a la herramienta en sí o si viene condicionada por otros elementos (p. ej. la temática que se abordaba, el grupo con el que se realizó, etc.).

\section{BIBLIOGRAFÍA}

Aguayo Vergara, M.; Bravo Molina, M.; Nocetti de la Barra, A.; Concha Sarabia, L. y Aburto Godoy, R. (2019): "Perspectiva estudiantil del modelo pedagógico flipped clasroom o aula invertida en el aprendizaje del inglés como lengua extranjera”, Revista Educación, vol. 43, ${ }^{\circ}$ 1. https://doi.org/10.15517/revedu.v43i1.31529.

Aguilera, C., Manzano, A., Martínez, I., Lozano, M.C y Casiano, C. (2017): “El modelo flipped Classroom", International Journal of Developmental and Educational Psychology(INFAD)Revista de Psicología, no 1-Monográfico 3, pp. 261-266.

Barkley, E.; Cross, K.P. y Major, C. (2007): Técnicas de aprendizaje colaborativo. Ministerio de Educación y Ciencia, Morata, Madrid.

Bergmann, J. y Sams, A. (2012): Flip your classroom: Reach every student in every class every day. Washington, DC: International Society for Technology in Education.

Bishop, J.L. y Verleger, M.A. (2013): “The flipped classroom: A survey of the research”. Paper presented at the ASEE Annual Conference y Exposition. Atlanta: American Society for Engineering Education.

Chen, Y.; Wang, Y. y Chen, N.S. (2014): "Is FLIP enough? Or should we use the FLIPPED model instead?”, Computers y Education, 79, pp. 16-27.

Davies, R.S.; Dean, D.L. y Ball, N. (2013): "Flipping the classroom and instructional technology integration in a college-level information systems spreadsheet course", Educational Technology Research and Development, 61(4), pp.563-580.

DeNeve, K. y Heppner, M. (1997). "Role play simulations: The assessment of an active learning technique and comparisons with traditional lectures”, Innovative Higher Education, vol. 21, no 3, pp. 231-246.

Enfield, J. (2013): "Looking at the impact of the flipped classroom model of instruction on undergraduate multimedia students at CSUN", TechTrends, vol. 57, $\mathrm{n}^{\circ}$ 6, pp. 14-27.

Fernández March, A. (2006): "Metodologías activas para la formación de competencias”, Educatio siglo XXI, n 24, pp. 35-56.

Fernández, A., Maiques, J.M., Ábalos, A. (2012): “Buenas prácticas docentes de los profesores universitarios: estudio de casos”, Revista de Docencia Universitaria (REDU), vol. 10, $\mathrm{n}^{\mathrm{o}}$ 1, pp.43-66.

Flores, O.; Del-Arco, I. y Silva, P. (2016): "The flipped classroom model at the university: analysis based on professors' and students' assessment in the educational field", International Journal of Educational Technology in Higher Education, pp.13-21.

Fortanet, C., González, G., Mira, R., y López, J. (2013): “Aprendizaje cooperativo y flipped classroom. Ensayos y resultados de la metodología docente”. En M. Teresa, D. Álvarez y N. Pellín (Presidencia), XI Jornadas de Redes de Investigación en Docencia Universitaria. Retos de futuro en la enseñanza superior: docencia e investigación para alcanzar la excelencia académica, Alicante.

Fulton, K.P. (2012): “10 reasons to flip”, Phi Delta Kappan, vol. 94, n² 2, pp. 20-24. 
Graaff, E. de y Bouhuijs, P. A. J. (eds.) (1993): Implementation of Problem based Learning in Higher Education, Thesis publishers, Amsterdam.

Gilboy, M.B.; Heinerichs, S. y Pazzaglia, G. (2015): "Enhancing student engagement using the flipped classroom”, Journal of Nutrition Education and Behavior, vol. 47, n 1, pp. 109114.

Gómez López, Y. y Muñoz Donate, F. (2018): Flipped Classroom en formación universitaria: aplicando una metodología inductiva para mejorar la eficacia del aprendizaje. Programa de formación de profesorado de la UNIA en materia de innovación docente y digital (2018-2019)

González-Gómez, D.; Jeong, J.S.; Airado-Rodríguez, D. y Cañada-Cañada, F. (2016): "Performance and perception in the Flipped Learning Model: An initial approach to evaluate the effectiveness of a new teaching methodology in a general science classroom”, Journal of Science Education and Technology, vol. 25, $\mathrm{n}^{\circ}$ 3, pp. 450-459.

Hommes, J., Keizer, P., Pettigrew, M. y Troy, J. (1999): Educational Innovation in Economics, Kluwer Academic Publisher, Boston.

Jones, N. F., Rassmussen, C. M. y Moffitt, M. C. (1997): Real-life problem solving: A collaborative approach to interdisciplinary learning, American Psychological Association, Washington.

Kapp, K.M. (2012): The gamification of Learning and Instruction: game based Methods and Strategies for Training and Education. Pfeiffer-Wiley, New York.

Khan, S. (2012): The one world schoolhouse: Education reimagined. London: Hodder and Stoughton.

Lago, C., Arce, E., Casaís, L., Fariña, F., Fernández, M., García, J. L. y Viaño, J. (2012): "Utilización de un sistema tecnológico de feedback interactivo en la docencia universitaria para mejorar el rendimiento académico del alumnado y la calidad docente del profesorado: El programa Turning Point”, Revista del CIDUI, 1. Disponible en http://www.cidui.org/revistacidui/ index.php/cidui/article/view/254

Lundin, M.; Rensfeldt, A.B.; Hillman, T.; Lantz-Andersson, A. y Peterson, L. (2018): "Higher education dominance and siloed knowledge: a systematic review of flipped classroom research”, International Journal of Educational Technology in Higher Education, vol. 15, $\mathrm{n}^{\mathrm{o}} 1$, pp. 15-20.

Martínez, W.; Esquivel, I. y Castillo, J. (2014): “Aula invertida o modelo invertido de aprendizaje: Origen, sustento e implicaciones” en Esquivel, I. (Coord.). Los modelos tecno-educativos, revolucionando el aprendizaje del siglo XXI, México, pp. 143-160.

Mason, G.S.; Shuman, T.R. y Cook, K.E. (2013): "Comparing the effectiveness of an inverted classroom to a traditional classroom in a upper-division engineering course”, IEEE Transactions and Education, vol. 56, $\mathrm{n}^{\circ}$ 4, pp. 430-435.

McCarthy, J. (2016): “Reflections on a flipped classroom in first year higher education”, Issues in Educational Research, vol. 26, $\mathrm{n}^{\circ}$ 2, pp. 332-350.

McGrath, D. (2002): "Teaching on the Front Lines: Using the Internet and Problem-Based Learning To Enhance Classroom Teaching”, Holist Nurs Pract, vol. 16, n 2, pp.5-13.

McLaughlin, J.E.; Griffin, L.M.; Esserman, D.A.; Davidson, C.A.; Glatt, D.M.; Roth, M.T. y Mumper, R.J. (2013): "Pharmacy student engagement, performance, and perception in a flipped satellite classroom”, American Journal of Pharmaceutical Education, vol. 77, $\mathrm{n}^{\circ}$ 9, article 196.

McLaughlin, J.E.; Roth, M.T.; Glatt, D.M.; Gharkolonaerehe, N.; Davidson, C.A.; Griffin, L.M.; Esserman, D.A. y Mumper, R.J. (2014): "The flipped classroom: A course redesign to foster learning and engagement in a health professions school”, Academic Medicine, vol. 89, n 2, pp. 236-243. 
McNally, B.; Chipperfield, J.; Dorsett, P.; Del Fabbro, L.; Frommolt, V.; Goetz, S.; Lewohl, J.; Molineux, M.; Pearson, A.; Reddan, G.; Roiko,A. y Rung, A. (2017): "Flipped classroom experiences: student preferences and flip strategy in a higher education context”, Higher Education, vol. 73, n 2, pp. 281-298.

Monedero, C.R., y Castro, A. (2018): "Un proyecto de aplicación de la clase invertida en la Ciencias de la Comunicación” en Redine (Ed.). Innovative Strategies For Higher Education in Spain, Adaya Press. pp. 69-79, Eindhoven, NL.

Moya Fuentes, M.M. y Soler García, C. (2018): "La gamificación mediante herramientas virtuales de respuesta de audiencia: la experiencia de Socrative y Kahoot!”, en RoigVila (ed). El compromiso académico y social a través de la investigación e innovación educativas en la Enseñanza Superior, Octaedro. pp. 1154-1163, Barcelona.

Murga-Menoyo, M.A, Bautista, M.J. y Novo, M. (2011): "Mapas conceptuales con Cmap Tools en la enseñanza universitaria de la educación ambiental. Estudio de caso en la UNED”, Enseñanza de las Ciencias, vol. 29, $\mathrm{n}^{\circ}$ 1, pp.47-60.

Nouri, J. (2016): "The flipped classroom for active, effective and increased learning-especially for low achievers", International Journal of Educational Technology in Higher Education, vol. 13, n ${ }^{\circ}$ 1, pp. 33.

Novak, J.D. y Cañas, A.J. (2006): La teoría subyacente a los mapas conceptuales y a cómo construirlos. Technical Report IHMC CmapTools 2006-01, Florida Institute for Human and Machine Cognition. http://www.ihmc.us/

Novak, J.D. y Gowin, D.B. (1988): Aprendiendo a Aprender. Ed. Martínez Roca: Barcelona.

O’Flaherty, J.; Phillips, C.; Karanicolas, S.; Snelling, C. y Winning, T. (2015): “The use of flipped classrooms in higher education: A scoping review”, The Internet and Higher Education, ${ }^{\circ}$ 25, pp. 85-95.

Pontes, A. (2012): "Representación y comunicación del conocimiento con mapas conceptuales en la formación del profesorado de ciencia y tecnología”, Revista Eureka sobre Enseñanza y Divulgación de las Ciencias, vol. 9, nº 1, pp. 106-123.

Prieto, A., Prieto, B., y Del Pino, B. (2016): “Una experiencia de flipped classroom”. En M. Torres y J. Cañadas (Presidencia), XXII Jornadas sobre la Enseñanza Universitaria de la Informática. Almería (España).

Roach, T. (2014): "Student perceptions toward flipped learning: New methods to increase interaction and active learning in economics", International Review of Economics Education, $\mathrm{n}^{\mathrm{0}}$ 17, pp. 74-84.

Rodríguez, K. P., Maya, M. A. y Jaén, J. S. (2012): “Educación en ingenierías: De las clases magistrales a la pedagogía del aprendizaje activo”, Revista Ingeniería y Desarrollo, vol. 30, $\mathrm{n}^{\circ} \quad 1, \quad$ pp. 125-142. Disponible en http://www.redalyc.org/articulo.oa?id=85223369008

Roehl, A.; Reddy, S.L. y Shannon, G.J. (2013): “The flipped classroom: An opportunity to engage millennial students through active learning strategies”, Journal of Family $y$ Consumer Sciences, vol. 105, $\mathrm{n}^{\circ}$ 2, pp. 44-49.

Sáez, B., Viñegla, S., y Piedad, M. (2014): “Una experiencia de flipped classroom”. En C. González, R. López, y J. M. Aroca (Ed), Educar para transformar. Actas XI Jornadas Internacionales de Innovación Universitaria, pp. 345-352, Universidad Europea de Madrid, Madrid.

Santiago, R. y Bergmann, J. (2018): Aprender al revés. Flipped learning 3.0 y metodologías activas en el aula, Paidós Educación, Barcelona

Sidhu, D.; Kwan, R.; Pexman, P. y Siakaluk, P. (2014): "Effects of relative embodiment in lexical and semantic processing of verbs”, Acta psychologica, $\mathrm{n}^{\circ}$ 149, pp. 32-39.

Strayer, J.F. (2012): "How learning in an inverted classroom influences cooperation, innovation and task orientation”, Learning Environments Research, vol. 15, n 2, pp. 171-193. 
The Flipped Classroom (2019). ¿Qué es flipped classroom? [Mensaje en un blog]. Recuperado de http://www.theflippedclassroom.es/what-is-innovacion-educativa/2019/02

Thomas, J. W. (2000): A review of research on project-based learning. California: Autodesk Foundation. Actualidadpedagogica.com "qué dicen los estudios sobre el aprendizaje basado en proyectos” José Sánchez. Disponible en: http://www.estuaria.es/wpcontent/uploads/2016/04/estudios_aprendizaje_basado_en_proyectos1.pdf

Tourón, J. y Santiago, R. (2015): "El modelo Flipped Learning y el desarrollo del talento en la escuela”, Revista de Educación, n ${ }^{0}$ 368, pp. 196-231.

Urbina, S., Arrabal, M., Conde, M., Ordinas, C., y Rodríguez, S. (2015): “Flipped classroom a través de videoconferencia. Un proyecto de innovación docente”, Campus Virtuales, vol. 4, $\mathrm{n}^{\circ}$ 2, pp. 60-65.

Vidal, M.D., Ferrón, V. y De la Torre, J.M. (2011): "La metodología del aprendizaje por indagación en la enseñanza universitaria: ejemplos concretos de aplicación”, Congreso Internacional de Innovación Docente, Cartagena (Murcia). 